\title{
Broad Spectrum Anti-Quorum Sensing Activity of Tannin-Rich Crude Extracts of Indian Medicinal Plants
}

\author{
Varsha Shukla ${ }^{1}$ and Zarine Bhathena ${ }^{2}$ \\ ${ }^{1}$ Department of Microbiology, Ramnarain Ruia College, Matunga, Mumbai 400019, India \\ ${ }^{2}$ Department of Microbiology, Bhavan's College, Andheri, Mumbai 400058, India \\ Correspondence should be addressed to Varsha Shukla; varshashukla@ruiacollege.edu
}

Received 25 December 2015; Revised 9 March 2016; Accepted 20 March 2016

Academic Editor: Giuseppe Comi

Copyright ( 2016 V. Shukla and Z. Bhathena. This is an open access article distributed under the Creative Commons Attribution License, which permits unrestricted use, distribution, and reproduction in any medium, provided the original work is properly cited.

\begin{abstract}
Quorum sensing (QS) mechanisms have been demonstrated to have significance in expression of pathogenicity in infectious bacteria. In Gram negative bacteria the autoinducer molecules that mediate QS are acyl homoserine lactones (AHL) and in Gram positive bacteria they are peptides called autoinducing peptides (AIP). A screening of tannin-rich medicinal plants was attempted to identify extracts that could interrupt the QS mechanisms in both Gram positive and Gram negative bacteria over a wide range of concentrations and therefore potentially be potent agents that could act as broad spectrum QS inhibitors. Six out of the twelve Indian medicinal plant extracts that were analyzed exhibited anti-QS activity in Chromobacterium violaceum 12472 and in S. aureus strain with agr:blaZ fusion over a broad range of subinhibitory concentrations, indicating that the extracts contain high concentration of molecules that can interfere with the QS mechanisms mediated by AHL as well as AIP.
\end{abstract}

\section{Introduction}

Unlike the early views on unicellular bacteria that suggested solitary behavior and little interactive capacity within a population, recent developments in ecological sciences suggest that communities, rather than individuals, play a major role in the maintenance of ecological stability $[1,2]$. Explorations for cooperative behavior in microbial communities have recently established that bacteria do communicate to coordinate the behavior of the population. One such phenomenon of organized social behavior in unicellular organisms is called quorum sensing (QS) [2]. Quorum sensing is a process of communication in bacteria via which bacteria are able to sense whether they have reached a density corresponding to their quorate population. At this density they are able to alternate gene expression such that the phenotype will be able to sustain the activities best suited for survival in the new environment [3]. QS takes place when the concentration of autoinducers released by bacteria reaches a critical threshold concentration, at which they bind to receptors and activate them to trigger genes that encode information associated with several characteristics, like bioluminescence, plasmid conjugation, biofilm formation, toxin production, exopolysaccharide production, siderophore synthesis, sporulation, and motility $[4,5]$, several of which determine pathogenicity of the organism. In Gram negative bacteria the autoinducing molecules are commonly $N$-acylated homoserine lactones (AHLs) whereas in Gram positives they are small peptides called autoinducing peptides (AIPs) [6]. QS in most Gram negative bacteria takes place via the LuxI/ LuxR system. luxI encodes an autoinducer synthase that catalyzes the formation of the signal molecule, AHL, that on reaching the quorum activates luxR that codes for the receptor of AHL. This complex then binds to the QS regulated promoters and leads to transcription of all genes controlled by QS system $[3,4,7,8]$. The best studied QS gene in Gram positive bacteria is the agr locus in Staphylococcus aureus that codes for its pathogenicity determinants. The agr locus consists of two divergent operons that are activated by promoters $\mathrm{P} 2$ and $\mathrm{P} 3$, respectively. $\mathrm{P} 2$ acts as a promoter for genes that synthesize the autoinducer. P3 codes for RNAIII, which is the regulatory effector of the agr regulon and initiates the transcription of genes that encode a variety of exoproteins responsible for pathogenicity [6, 9-11].

Because QS controls the virulons in many infectious bacteria like Vibrio cholerae, Escherichia coli, Bacillus cereus 
[6, 12], Pseudomonas aeruginosa, Staphylococcus aureus [3], and Acinetobacter baumannii [13], blocking of this mechanism would prevent pathogenicity and hence attenuate the infectious agent [12]. Quorum sensing inhibitors (QSI) can be excellent antipathogenic agents that will not interfere with growth but can prevent pathological consequences. Virulence determinants in pathogens are not strictly essential for viability. Thus anti-QS compounds will not induce mutagenesis that can lead to the emergence of resistance. Such agents will instead promote clearance of the pathogen by stimulating the immune mechanisms with live, multiplying organisms for a longer duration [14]. Rasmussen and Givskov (2006) [15] have suggested that quorum sensing can be attacked at three points: either by blocking production of the autoinducer, by inactivating it, or by interfering in its binding to the receptor.

In the recent years, screening for anti-QS substances in eukaryotes like plants, algae, and fungi is gaining significance. As these eukaryotes do not possess active immune systems, they rely on physical and chemical defense mechanisms. It is therefore hypothesized that secondary metabolites produced by them can aid in preventing colonization by pathogens. Such natural quorum sensing interfering compounds are reported in exudates of several vegetables and spices and are also found to be secreted by Penicillium spp. and Chlamydomonas reinhardtii $[4,14-17]$.

Considering the enormous number of plant varieties and the chemical diversity that plants inherently possess, screening plants for medicinally significant compounds seems rational. Various medicinal plants have been screened for their anti-QS potential [18-22], and several phytochemicals have been shown to affect expression of pathogenicity via disruption of QS [23-27]. The current study uses an ethnobotanical approach and focuses on anti-QS properties of tannin-rich extracts of plants used in Indian traditional and folk medicine, as plants rich in tannins are naturally protected from predation and pathogens. Antimicrobial, antiviral, and anticancer activities of tannins have been reported [28, 29]. Anti-AHL activity of synthetic tannic acid was reported by Huber et al. (2003) [30]. Recently some reports on purified tannin-rich components exhibiting either anti-AHL or antiagr activity have also been made [31-34]. In the current study, we have quantified both anti-AHL and anti-agr activity of tannin-rich plant extracts with the aim of determining spectrum of activity and of suggesting their potencies as antiQS substances.

\section{Materials and Methods}

2.1. Preparation of Plant Extracts. Powders of seeds of Syzygium cumini and Embelia ribes were acquired from the local market. Fruits of Phyllanthus emblica, Terminalia bellirica, and Terminalia chebula; pericarp of Punica granatum; flowers and seed kernel of Mangifera indica; and the barks of Acacia arabica, Terminalia arjuna, Thespesia populnea, and Casuarina equisetifolia were collected from Mumbai and the respective plant samples were authenticated at Agharkar Institute, Pune, and The Blatter Herbarium, St. Xavier's College, Mumbai. The plant parts were washed, dried, and powdered. Methanolic extracts were prepared by soaking the powders
(1 gm) in methanol $(10 \mathrm{~mL})$ overnight. These extracts were used for phytochemical screening. For the quantitative biological assays the dried methanolic extracts were dissolved in $7.8 \%$ DMSO to known concentrations and stored at $-25^{\circ} \mathrm{C}$ [35].

2.2. Phytochemical Screening of Extracts. Extracts were qualitatively analyzed for presence of phytochemicals, namely, saponins, alkaloids, flavonoids, di- and triterpenes, phenols, and tannins using methods suggested by Roopashree et al. (2008) [36]. The acid butanol test was used to determine whether the tannins were of the condensed type [37].

2.3. Bacterial Strains. Three biomonitor strains were used for anti-AHL activity:

(a) Chromobacterium violaceum ATCC 12472, wild type strain that produces a purple pigment in response to QS (C6 AHL) molecules that it produces;

(b) Chromobacterium violaceum ATCC 31532, nonpigmenting strain that overproduces C6-AHL, the autoinducer;

(c) Chromobacterium violaceum 026, a mini Tn5 mutant of Chromobacterium violaceum 31532, which is unable to produce the autoinducer C6-AHL but responds to an exogenous supply of C6-AHL by producing the purple pigment [38].

All three strains were maintained on Luria agar.

Biomonitor strain Staphylococcus aureus agr P3::blaZ RN6390 pRN8826 that contains agrP3-blaZ fusion plasmid and produces $\beta$-lactamase spontaneously due to agr expression was used for detection of anti-QS activity that targeted AIP mediated QS. This strain was maintained on Luria agar with $10 \mu \mathrm{g} / \mathrm{mL}$ chloramphenicol.

2.4. Determination of Minimum Inhibitory Concentration (MIC) of Extracts against Biomonitor Strains. MICs of the extracts were determined essentially to decide subinhibitory concentrations (SICs) that were used for quantitative antiQS activity in Gram positive and Gram negative biosensor strains. MIC was determined using the microdilution method given by Valgas et al. (2007) [39]. $8 \mathrm{mg} / \mathrm{mL}$ of methanolic extract in 7.8\% DMSO of each was diluted twofold in Luria broth to a total volume of $100 \mu \mathrm{L}$ in 96-well microtitre plates. Wells were inoculated with $5 \mu \mathrm{L}$ of a bacterial suspension $\left(10^{8} \mathrm{CFU} / \mathrm{mL}\right)$ of the C. violaceum $12472 / S$. aureus agr P3::blaZ RN6390. All experiments were performed in triplicate and plates were incubated at $37^{\circ} \mathrm{C}$ for $24 \mathrm{~h}$. After incubation $50 \mu \mathrm{L}$ of $0.2 \mathrm{mg} / \mathrm{mL}$ alcoholic solution of INT (2-(4-iodophenyl)-3-(4-nitrophenyl)-5-phenyl-2Htetrazolium) (Hi media) was added and plates were incubated at $37^{\circ} \mathrm{C}$ for $30 \mathrm{~min}$. Reduction of INT to its formazan product was a clear indication of growth/no growth. Change in color to pink was noted as growth. Concentrations lower than the cidal concentration were then used as SICs. 


\subsection{Anti-QS Activity of Plant Extracts Using Gram Negative Biomonitor Strain}

2.5.1. Quantitative Studies. For quantitative studies of the anti-QS activity, a modified method of Blosser and Gray (2000) [40] was used. This assay estimated the pigment produced by C. violaceum ATCC 12472. Concentrations of the extracts that were selected were below the MIC and equaled $0.5 \mathrm{MIC}(\mathrm{N} / 2), 0.25 \mathrm{MIC}(\mathrm{N} / 4), 0.125 \mathrm{MIC}(\mathrm{N} / 8)$, $0.0625 \mathrm{MIC}(\mathrm{N} / 16)$, and $0.03125 \mathrm{MIC}(\mathrm{N} / 32)$ of each extract and were prepared in Luria broth. To $1 \mathrm{~mL}$ of each SIC $50 \mu \mathrm{L}$ of overnight culture $\left(10^{7} \mathrm{cfu} / \mathrm{mL}\right)$ of $C$. violaceum ATCC 12472 was added and tubes were incubated for 24 hours at room temperature. Medium without the extract served as a positive control, medium with 7.8\% DMSO served as vehicle control, and medium with subinhibitory concentrations of extract but no culture served as blanks. After incubation, amount of growth in each tube was accessed by measuring absorbance at $655 \mathrm{~nm}$. The pigment produced was extracted by adding equal quantity of acetone to the harvested biomass [41] and absorbance was measured at $567 \mathrm{~nm}$ on Jasco V363 spectrophotometer against each blank. Percentage of pigment formed was calculated with respect to the vehicle control. All assays were performed in triplicate and average percent pigmentation values were reported with SD that was calculated using the Microsoft EXCEL software. Minimum concentration in $\mathrm{mg} / \mathrm{mL}$ of plant extract, at which $50 \%$ reduction in pigment formation was observed, was designated as the quorum sensing inhibitory concentration (MQSIC) [31].

2.5.2. Determination of luxR/luxI Effect. To determine whether inhibition of QS was due to the effect on $N$-acyl homoserine lactone response (luxR effect) or due to its effect on synthesis of AHL (luxI effect), method of Mihalik et al. (2008) [42] was used. Discs of Whatman filter paper number 1 with $75 \mu \mathrm{L}$ of methanolic plant extracts were placed on Luria agar plates. C. violaceum strains, CV026 and CV31532, were streaked in circles $8-10 \mathrm{~mm}$ apart such that in one plate strain CV026 formed the inner circle and strain CV31532 the outer circle, while in the other plate it was vice versa. On incubation at RT for 24 hours pigmentation in the CV026 strain was used to determine the luxR/luxI effect. A lowered signal in the form of pigmentation of CV026 when it forms the outer circle suggested that LuxI was the target while a lowered signal in the form of pigmentation of CV026 when it forms the inner circle suggested that LuxR was the target.

2.6. Anti-QS Activity of Plant Extracts Using Gram Positive Biosensor Strain. Quantitative measurement of agr activity was done using the $S$. aureus agr $\mathrm{P} 3$ ::blaZ reporter assay, originally described by Ji et al. (1995) [43]. This assay measured RNA III production in the form of $\beta$-lactamase activity using the chromogenic cephalosporin, nitrocefin, as a substrate. $S$. aureus agr P3-blaZ strain was grown in CYGP broth overnight at $37^{\circ} \mathrm{C}$. The overnight broth culture was further diluted $1 / 100$ with fresh medium and incubated at $37^{\circ} \mathrm{C}$ to attain logarithmic phase $\left(A_{600}, 0.4\right)$. In a microtitre plate, $5 \mu \mathrm{L}$ of tannin-rich plant extract prepared in CYGP broth was added to $45 \mu \mathrm{L}$ of $\log$ phase culture such that it reached a subinhibitory concentration equivalent to $0.5 \mathrm{MIC}(\mathrm{N} / 2), 0.25 \mathrm{MIC}$ $(N / 4), 0.125 \mathrm{MIC}(\mathrm{N} / 8), 0.0625 \mathrm{MIC}(\mathrm{N} / 16)$, and $0.03125 \mathrm{MIC}$ $(N / 32)$. In the positive control well $5 \mu \mathrm{L}$ of supernatant of the overnight broth culture was added as a source of AIP to $45 \mu \mathrm{L}$ of the log phase culture, whereas $5 \mu \mathrm{L}$ of $7.8 \%$ DMSO was added to $45 \mu \mathrm{L}$ of the log phase culture as vehicle control. The plate was incubated at $37^{\circ} \mathrm{C}$ on Selec microplate shaker for 55 minutes. After incubation $50 \mu \mathrm{L}$ of CYGP broth containing $5 \mathrm{mM}$ sodium azide was added, followed by $50 \mu \mathrm{L}$ of $132 \mu \mathrm{g} / \mathrm{mL}$ nitrocefin (Toku-E) prepared in $0.1 \mathrm{M}$ sodium phosphate buffer, $\mathrm{pH} 5.8$. $\beta$-lactamase activity was measured in Biorad imark microplate reader using kinetic mode at $\varepsilon 490-\varepsilon 650 \mathrm{~nm}$. One unit of $\beta$-lactamase activity was defined as an increase in $0.001 \mathrm{OD}$ units per min. All assays were performed in triplicate and average percent enzyme activity was calculated with respect to the vehicle control. \% enzyme activity values were reported with SD that was calculated using the Microsoft EXCEL software. Minimum concentration of extract in $\mathrm{mg} / \mathrm{mL}$ that showed $50 \%$ inhibition in activity was determined and designated as the minimum quorum sensing inhibitory concentration (MQSIC) [32].

\section{Results and Discussion}

3.1. Phytochemical Screening. All the methanolic extracts when screened for their phytochemical constituents showed strong reactions with $\mathrm{FeCl}_{3}$. The protein precipitation test too was strongly positive for all extracts. This confirmed that all the shortlisted plants were rich in tannins. Tannins are primarily of two types: condensed and hydrolysable. Qualitative analysis for condensed tannins showed that the extracts of Acacia arabica, Terminalia arjuna, Casuarina equisetifolia, Thespesia populnea, Mangifera indica (seed kernel), and Embelia ribes were rich in condensed tannins. In contrast, extracts of Phyllanthus emblica, Terminalia bellirica, Terminalia chebula, Punica granatum and Mangifera indica (flower), and Syzygium cumini were rich in hydrolysable tannins as they showed high tannin content but gave a negative acid butanol test [44-49].

\subsection{Anti-QS Activity of Plant Extracts Using Gram Negative Biomonitor Strain (Anti-AHL Activity)}

3.2.1. Quantitative Studies. Amount of pigment produced by C. violaceum 12472 on exposure to subinhibitory concentrations of extracts showed an increase with decreasing concentration of extract. All the twelve extracts showed anti-QS activity at subinhibitory concentrations (Table 1). All the extracts showed not less than $65 \%$ growth in $N / 2$ concentration, not less than $82 \%$ in N/4 concentration, and between 85 and $114 \%$ thereafter with respect to the vehicle control, indicating that the SICs used did not significantly affect cell density. A comparison of MQSIC with the MIC of the extracts (Figure 1), therefore, clearly depicted the range of concentration where the extracts had a specific and optimal QS inhibition activity with zero or minimal effect on growth of $C$. violaceum. Extracts of $P$. granatum, S. cumini, and T. chebula 
TABLE 1: Quantitative studies of anti-quorum sensing activity detected as pigmentation in C. violaceum ATCC 12472 in the presence of subinhibitory concentrations of plant extracts.

\begin{tabular}{|c|c|c|c|c|c|}
\hline \multirow{2}{*}{ Plant } & \multicolumn{5}{|c|}{$\%$ pigmentation with respect to untreated control $(N=\mathrm{MIC}$ in $\mathrm{mg} / \mathrm{mL})$} \\
\hline & $N / 2$ & $N / 4$ & $N / 8$ & $N / 16$ & $N / 32$ \\
\hline P. emblica & $7.29 \pm 12.36$ & $29.58 \pm 8.66$ & $41.70 \pm 6.19$ & $66.50 \pm 2.82$ & $102.84 \pm 8.94$ \\
\hline T. bellirica & $0.57 \pm 1.00$ & $19.66 \pm 8.79$ & $28.54 \pm 8.9$ & $56.63 \pm 6.01$ & $68.39 \pm 12.13$ \\
\hline T. chebula & 0.00 & $2.02 \pm 2.93$ & $21.22 \pm 4.61$ & $47.85 \pm 5.40$ & $72.18 \pm 9.45$ \\
\hline P. granatum & $20.08 \pm 6.78$ & $23.21 \pm 7.86$ & $25.75 \pm 8.54$ & $32.17 \pm 3.49$ & $72.05 \pm 11.26$ \\
\hline S. cumini & $0.72 \pm 0.66$ & $5.64 \pm 5.32$ & $12.88 \pm 7.72$ & $28.70 \pm 6.86$ & $78.53 \pm 19.27$ \\
\hline M. indica (flower) & $6.48 \pm 0.42$ & $28.59 \pm 12.61$ & $34.19 \pm 12.08$ & $72.33 \pm 6.49$ & $87.05 \pm 8.43$ \\
\hline E. ribes & $13.34 \pm 6.21$ & $30.60 \pm 6.02$ & $51.19 \pm 3.83$ & $89.49 \pm 14.54$ & $90.18 \pm 4.00$ \\
\hline A. arabica & $5.56 \pm 3.52$ & $42.50 \pm 7.11$ & $56.68 \pm 2.25$ & $109.21 \pm 31.91$ & $119.58 \pm 13.93$ \\
\hline M. indica (seed) & $4.83 \pm 1.76$ & $47.05 \pm 3.20$ & $61.47 \pm 8.58$ & $76.55 \pm 17.50$ & $87.87 \pm 4.97$ \\
\hline T. arjuna & $3.20 \pm 2.86$ & $46.26 \pm 4.52$ & $71.40 \pm 15.54$ & $88.89 \pm 15.45$ & $96.54 \pm 3.83$ \\
\hline C. equisetifolia & $6.93 \pm 2.54$ & $16.85 \pm 3.72$ & $59.94 \pm 10.98$ & $89.43 \pm 11.55$ & $86.55 \pm 13.10$ \\
\hline T. populnea & $0.01 \pm 0.01$ & $10.14 \pm 9.74$ & $56.16 \pm 9.55$ & $68.72 \pm 11.66$ & $80.09 \pm 8.92$ \\
\hline
\end{tabular}

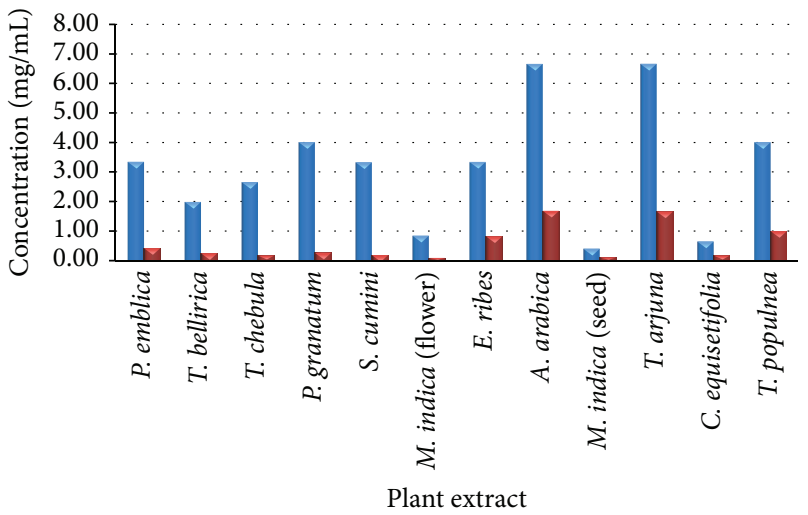

$\because \mathrm{MIC}$

- MQSIC

FIgURE 1: Comparison of MIC values with MQSIC values for antiquorum sensing activity of plant extracts, detected using biosensor strain C. violaceum 12472.

were most significant as there was a marked difference (1/16) in their MIC and MQSIC. In contrast, extracts of E. ribes, $A$. arabica and $M$. indica (seed), T. arjuna, C. equisetifolia, and T. populnea had a very low range of concentration at which they could prevent QS, as the difference between their MIC and MQSIC was only 4 -fold, while extracts of P. emblica, T. bellirica, and $M$. indica showed N/8 as their MQSIC value.

3.2.2. luxI/luxR Effect. To investigate whether QS inhibition might be due to interference with acyl homoserine lactone production (luxI effect) or a transcription response (luxR effect) the luxI/luxR assay was done. Amongst the hydrolysable tannin-rich extracts studied T. bellirica, T. chebula, S. cumini, and $M$. indica (seed) showed a putative luxI effect, indicating that they would affect production of the C6 HSL by CV31532, whereas P. emblica showed a putative luxR effect indicating that it would interfere with the binding of the autoinducer to receptors on CV026. It is interesting to note
TABLE 2: Demonstration of luxI/luxR effect by plant extracts.

\begin{tabular}{lcc}
\hline Extract & luxI effect & luxR effect \\
\hline P. emblica & - & + \\
T. bellirica & + & - \\
T. chebula & + & - \\
P. granatum & + & + \\
S. cumini & + & - \\
M. indica (flower) & + & + \\
E. ribes & - & - \\
A. arabica & - & - \\
M. indica (seed) & + & - \\
T. arjuna & - & - \\
C. equisetifolia & - & - \\
T. populnea & - & - \\
\hline
\end{tabular}

that $P$. granatum and $M$. indica (flower) showed both luxI and luxR effect (Table 2 and Figure 2).

3.3. Anti-QS Activity of Plant Extracts Using Gram Positive Biosensor Strain (Anti-agr Activity). $\beta$-lactamase produced as a result of QS in biomonitor strain $S$. aureus agrP3::blaZ RN6390 pRN8826 was estimated using chromogenic cephalosporin that on breakdown by $\beta$-lactamase gave a red colored product. Estimation of the enzyme units of $\beta$ lactamase was used as a measure of agr activity and, hence, QS in the S. aureus agrP3::blaZ strain (Table 3). The most significant extract amongst those tested was T. chebula as it showed only about $30 \%$ activity at the lowest subinhibitory concentration tested that is 0.03125 of its MIC. S. cumini, P. granatum, and $M$. indica (seed) too showed low activity at this concentration. A comparison of the MQSIC with the MIC of the extracts (Figure 3) was used as a representation of the range of concentration, where the extracts had a specific and optimal QS inhibition activity with zero or minimal effect on growth of $S$. aureus agrP3::blaZ RN6390. The MQSIC in $P$. emblica, T. bellirica, P. granatum, and M. indica (seed) was 16 


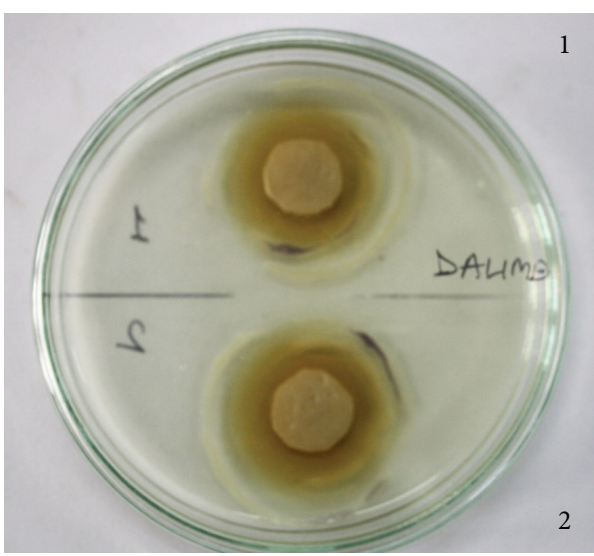

(a)

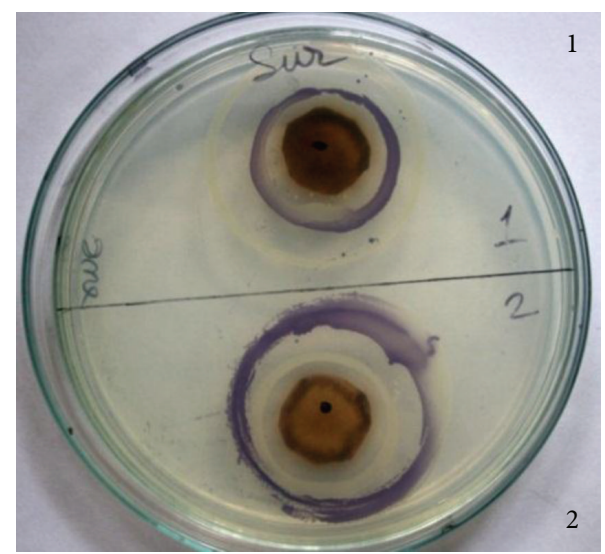

(b)

FIGURE 2: luxI and $l u x R$ effect: extract prepared from P. granatum affects production as well as activity of the autoinducer (a). Extract prepared from C. equisetifolia is unable to affect either production or binding of the autoinducer (b) (1: luxR effect, 2: luxI effect).

TABLE 3: Quantitative studies of anti-quorum sensing activity detected as $\beta$-lactamase activity in biosensor strain S. aureus agrP3::blaZ in the presence of subinhibitory concentrations of plant extracts.

\begin{tabular}{|c|c|c|c|c|c|}
\hline \multirow{2}{*}{ Plant } & \multicolumn{5}{|c|}{$\% \beta$-lactamase activity with respect to untreated control $(N=$ MIC in $\mathrm{mg} / \mathrm{mL})$} \\
\hline & $N / 2$ & $N / 4$ & $N / 8$ & $N / 16$ & $N / 32$ \\
\hline P. emblica & $20.9 \pm 7.87$ & $37.9 \pm 2.43$ & $34.5 \pm 3.25$ & $39.1 \pm 2.47$ & $63.6 \pm 13.08$ \\
\hline T. bellirica & $16.3 \pm 2.45$ & $5.6 \pm 2.11$ & $36.9 \pm 3.55$ & $43.5 \pm 4.33$ & $80.2 \pm 6.32$ \\
\hline T. chebula & $7.5 \pm 5.85$ & $18.9 \pm 2.69$ & $24.7 \pm 5.70$ & $28.4 \pm 9.48$ & $30.2 \pm 4.18$ \\
\hline P. granatum & $14.6 \pm 5.13$ & $21.3 \pm 2.54$ & $20.8 \pm 6.26$ & $33.2 \pm 7.79$ & $51.1 \pm 11.24$ \\
\hline S. cumini & $6.5 \pm 2.12$ & $22.4 \pm 0.77$ & $28.1 \pm 5.06$ & $39.6 \pm 5.25$ & $46.0 \pm 4.07$ \\
\hline M. indica (flower) & 0.0 & 0.0 & $80.3 \pm 9.71$ & $82.7 \pm 10.71$ & $82.3 \pm 14.53$ \\
\hline E. ribes & $57.4 \pm 16.45$ & $65.8 \pm 5.28$ & $65.0 \pm 7.00$ & $70.3 \pm 9.27$ & $97.7 \pm 13.19$ \\
\hline A. arabica & $33.0 \pm 1.97$ & $32.5 \pm 6.34$ & $34.5 \pm 4.61$ & $55.2 \pm 3.72$ & $61.8 \pm 2.65$ \\
\hline M. indica (seed) & 0.0 & $28.4 \pm 10.25$ & $53.8 \pm 2.27$ & $52.4 \pm 4.77$ & $54.6 \pm 1.87$ \\
\hline T. arjuna & $52.6 \pm 9.95$ & $53.7 \pm 15.32$ & $50.2 \pm 1.64$ & $52.3 \pm 3.46$ & $60.6 \pm 6.83$ \\
\hline C. equisetifolia & $37.0 \pm 8.53$ & $38.3 \pm 7.22$ & $59.7 \pm 2.46$ & $64.8 \pm 9.49$ & $64.4 \pm 6.56$ \\
\hline T. populnea & $13.0 \pm 8.80$ & $22.7 \pm 2.84$ & $38.5 \pm 3.59$ & $55.5 \pm 1.22$ & $57.8 \pm 6.28$ \\
\hline
\end{tabular}

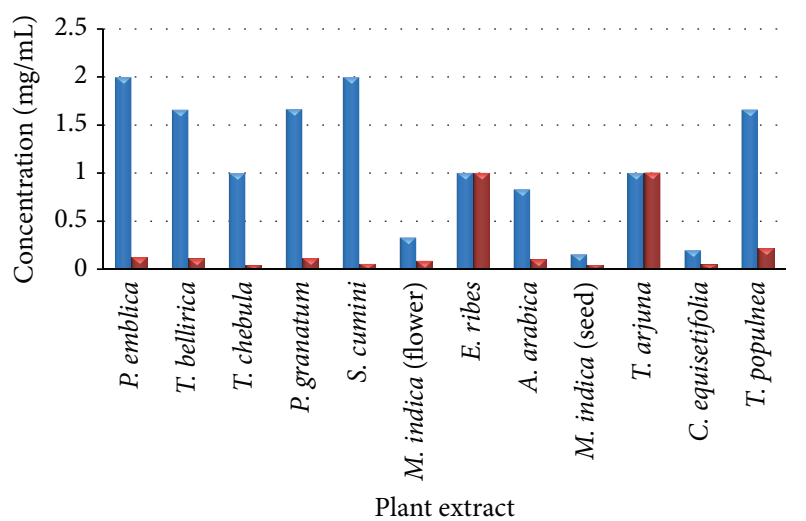

- MIC

- MQSIC

FIGURE 3: Comparison of MIC values with MQSIC values for antiquorum sensing activity of plant extracts, detected using biosensor strain S. aureus agrP3::blaZ. times lower than its MIC and in T. populnea and A. arabica it was found to be 8 times lesser, while in C. equisetifolia it was 4 times lesser than its MIC.

In a qualitative study, Shukla and Bhathena (2014) [50] have reported that several tannin-rich crude extracts show a broad spectrum of anti-QS activity. Another qualitative study on anti-AHL activity of pericarp of Punica granatum and an ethyl acetate fraction of $S$. cumini leaves has also been reported [22, 51]. Hasan et al. (2012) [23] have reported that $P$. emblica fruit extract can suppress quorum sensing genes in $S$. mutans. These reports draw attention to the fact that tanninrich extracts of medicinal plants influence quorum sensing mechanisms.

The current study is a quantitative evaluation of antiAHL and anti-agr activity of tannin-rich plants. One of the striking inferences of this study is that extracts rich in condensed tannins are unable to prevent AHL as well as agr mediated bacterial communication over a wide range of subinhibitory concentrations. On the other hand, extracts 
rich in hydrolysable tannins, namely, P. emblica, T. bellirica, T. chebula, P. granatum, S. cumini, and M. indica (flower), exhibit a broad spectrum anti-QS activity, that is, affecting activity of acyl homoserine lactones as well as autoinducers over a wide range of subinhibitory concentrations. Okuda and Ito (2011) [52] have attributed most of the biological and pharmacological activities of tannins determined so far to the simpler tannins like hydrolysable tannins. Among the extracts rich in hydrolysable tannins, T. chebula, P. granatum, and S. cumini can be categorized as potent quorum sensing inhibitors as they demonstrate this activity at concentrations that are several times lower than their respective MIC values. Anti-AHL activity of pericarp of Punica granatum using a qualitative method was reported by Zahin et al. (2010) [22]. The current study goes one step further to show that $P$. granatum is effective over a wide range of concentrations much lesser than its MIC and also that it affects AHL activity by influencing both production and its binding. The anti-QS property of $P$. granatum gets augmented, as it also shows significant anti-agr activity. Since all the extracts show distinct protein binding ability, these extracts may be disrupting QS either by inactivating enzymes responsible for the synthesis of the autoinducers or by binding to protein receptors of QS signals as demonstrated in the luxI/luxR assay.

\section{Conclusion}

Although the discovery of antibiotics has been a boon to human civilization, the current traditional antimicrobials need to be reviewed for their efficacy because of the increasing occurrence of multidrug resistant strains. One novel therapeutic approach to overcome the problem of resistance is the use of antipathogenic drugs that target key regulatory bacterial systems responsible for the expression of virulence factors. Since quorum sensing leads to expression of virulence genes in several known pathogens, agents that can interrupt bacterial communication can be used as antipathogenic drugs. The study on tannin-rich crude extracts from Indian medicinal plants used in Ayurveda showed that extracts of hydrolysable tannin-rich plants, Phyllanthus emblica, Terminalia bellirica, Terminalia chebula, Punica granatum, Syzygium cumini, and Mangifera indica (flower), are effective QSIs that can interrupt QS in both Gram positive and Gram negative bacteria over a wide range of subinhibitory concentrations, suggesting the presence of a high concentration of anti-QS molecules. Thus plant extracts rich in hydrolysable tannins can be used as broad spectrum antipathogenic drugs at concentrations that will not impose selection pressure. As this study has been done on crude extracts, purification and identification of the active principle along with verification of its anti-QS activity using in vivo studies are essential. However, such a pharmacognosy approach to the so-called alternative systems of medicine can be of immense help in the search for novel phytocompounds with medical applications.

\section{Competing Interests}

The authors declare that there is no conflict of interests regarding the publication of this paper.

\section{Acknowledgments}

Chromobacterium violaceum ATCC 12472, Chromobacterium violaceum ATCC 31532, and Chromobacterium violaceum 026 were kindly supplied by Dr. Robert McClean of Department of Biology, Texas State University. Biosensor strain for detection of QSI that affects AIP mediated quorum sensing, $S$. aureus agr P3::blaZ RN6390 pRN8826, was kindly supplied by Dr. Richard Novick, New York University.

\section{References}

[1] C. Gera and S. Srivastava, "Quorum-sensing: the phenomenon of microbial communication," Current Science, vol. 90, no. 5, pp. 666-676, 2006.

[2] I. Joint, J. A. Downie, and P. Williams, "Bacterial conversations: talking, listening and eavesdropping. An introduction," Philosophical Transactions of the Royal Society of London, Series B: Biological Sciences, vol. 362, no. 1483, pp. 1115-1117, 2007.

[3] S. Swift, M. C. Rowe, and M. Kamath, "Quorum sensing," in Bacterial Phisiology-A Molecular Approach, W. El-Sharoud, Ed., pp. 179-232, Springer, Berlin, Germany, 2008.

[4] J. E. González and N. D. Keshavan, "Messing with bacterial quorum sensing," Microbiology and Molecular Biology Reviews, vol. 70, no. 4, pp. 859-875, 2006.

[5] D. Ren, R. Zuo, A. F. G. Barrios et al., "Differential gene expression for investigation of Escherichia coli biofilm inhibition by plant extract ursolic acid," Applied and Environmental Microbiology, vol. 71, no. 7, pp. 4022-4034, 2005.

[6] L. C. M. Antunes, R. B. R. Ferreira, M. M. C. Buckner, and B. B. Finlay, "Quorum sensing in bacterial virulence," Microbiology, vol. 156, no. 8, pp. 2271-2282, 2010.

[7] P. Williams, "Quorum sensing, communication and cross-kingdom signalling in the bacterial world," Microbiology, vol. 153, no. 12, pp. 3923-3938, 2007.

[8] J. T. Hodgkinson, M. Welch, and D. R. Spring, "Learning the language of bacteria," ACS Chemical Biology, vol. 2, no. 11, pp. 715-717, 2007.

[9] R. P. Novick and E. Geisinger, "Quorum sensing in staphylococci," Annual Review of Genetics, vol. 42, pp. 541-564, 2008.

[10] R. O. Jensen, K. Winzer, S. R. Clarke, W. C. Chan, and P. Williams, "Differential recognition of Staphylococcus aureus quorum-sensing signals depends on both extracellular loops 1 and 2 of the transmembrane sensor AgrC," Journal of Molecular Biology, vol. 381, no. 2, pp. 300-309, 2008.

[11] N. Harraghy, S. Kerdudou, and M. Herrmann, "Quorum-sensing systems in staphylococci as therapeutic targets," Analytical and Bioanalytical Chemistry, vol. 387, no. 2, pp. 437-444, 2007.

[12] S. T. Rutherford and B. L. Bassler, "Bacterial quorum sensing: its role in virulence and possibilities for its control," Cold Spring Harbor Perspectives in Medicine, vol. 2, no. 11, 2012.

[13] Y.-S. Kang and W. Park, "Contribution of quorum-sensing system to hexadecane degradation and biofilm formation in Acinetobacter sp. strain DR1," Journal of Applied Microbiology, vol. 109, no. 5, pp. 1650-1659, 2010.

[14] M. Hentzer and M. Givskov, "Pharmacological inhibition of quorum sensing for the treatment of chronic bacterial infections," Journal of Clinical Investigation, vol. 112, no. 9, pp. 13001307, 2003. 
[15] T. B. Rasmussen and M. Givskov, "Quorum sensing inhibitors: a bargain of effects,” Microbiology, vol. 152, no. 4, pp. 895-904, 2006.

[16] T. Bjarnsholt, P. Ø. Jensen, T. B. Rasmussen et al., "Garlic blocks quorum sensing and promotes rapid clearing of pulmonary Pseudomonas aeruginosa infections," Microbiology, vol. 151, no. 12, pp. 3873-3880, 2005.

[17] T. B. Rasmussen, T. Bjarnsholt, M. E. Skindersoe et al., "Screening for Quorum-Sensing Inhibitors (QSI) by use of a novel genetic system, the QSI selector," Journal of Bacteriology, vol. 187, no. 5, pp. 1799-1814, 2005.

[18] C.-L. Koh, C.-K. Sam, W.-F. Yin et al., "Plant-derived natural products as sources of anti-quorum sensing compounds," Sensors, vol. 13, no. 5, pp. 6217-6228, 2013.

[19] A. Adonizio, K.-F. Kong, and K. Mathee, "Inhibition of quorum sensing-controlled virulence factor production in Pseudomonas aeruginosa by South Florida plant extracts," Antimicrobial Agents and Chemotherapy, vol. 52, no. 1, pp. 198-203, 2008.

[20] D. Damte, E. Gebru, S.-J. Lee, J.-W. Suh, and S.-C. Park, "Evaluation of anti-quorum sensing activity of 97 indigenous plant extracts from korea through bioreporter bacterial strains chromobacterium violaceum and pseudomonas aeruginosa," Journal of Microbial and Biochemical Technology, vol. 5, no. 2, pp. 42-46, 2013.

[21] S. S.-M. Yeo and F.-Y. Tham, "Anti-quorum sensing and antimicrobial activities of some traditional chinese medicinal plants commonly used in South-East Asia," Malaysian Journal of Microbiology, vol. 8, no. 1, pp. 11-20, 2012.

[22] M. Zahin, S. Hasan, F. Aqil, M. S. A. Khan, F. M. Husain, and I. Ahmad, "Screening of certain medicinal plants from India for their anti-quorum sensing activity," Indian Journal of Experimental Biology, vol. 48, no. 12, pp. 1219-1224, 2010.

[23] S. Hasan, M. Danishuddin, M. Adil, K. Singh, P. K. Verma, and A. U. Khan, "Efficacy of E. officinalis on the cariogenic properties of Streptococcus mutans: a novel and alternative approach to suppress Quorum-sensing mechanism," PLoS ONE, vol. 7, no. 7, Article ID e40319, 2012.

[24] F. M. Husain, I. Ahmad, M. S. Khan, and N. A. Al-Shabib, "Trigonella foenum-graceum (Seed) extract interferes with quorum sensing regulated traits and biofilm formation in the strains of Pseudomonas aeruginosa and Aeromonas hydrophila," Evidence-Based Complementary and Alternative Medicine, vol. 2015, Article ID 879540, 10 pages, 2015.

[25] F. Nazzaro, F. Fratianni, and R. Coppola, "Quorum sensing and phytochemicals," International Journal of Molecular Sciences, vol. 14, no. 6, pp. 12607-12619, 2013.

[26] O. M. Vandeputte, M. Kiendrebeogo, T. Rasamiravaka et al., "The flavanone naringenin reduces the production of quorum sensing-controlled virulence factors in pseudomonas aeruginosa PAO1," Microbiology, vol. 157, no. 7, pp. 2120-2132, 2011.

[27] H. Yin, Y. Deng, H. Wang, W. Liu, X. Zhuang, and W. Chu, “Tea polyphenols as an antivirulence compound disrupt quorumsensing regulated pathogenicity of Pseudomonas aeruginosa," Scientific Reports, vol. 5, Article ID 16158, 2015.

[28] J. Dai and R. J. Mumper, "Plant phenolics: extraction, analysis and their antioxidant and anticancer properties," Molecules, vol. 15, no. 10, pp. 7313-7352, 2010.

[29] M. M. Cowan, "Plant products as antimicrobial agents," Clinical Microbiology Reviews, vol. 12, no. 4, pp. 564-582, 1999.

[30] B. Huber, L. Eberl, W. Feucht, and J. Polster, "Influence of polyphenols on bacterial biofilm formation and quorum-sensing,"
Zeitschrift fur Naturforschung-Section C, vol. 58, no. 11-12, pp. 879-884, 2003.

[31] J. C. Taganna, J. P. Quanico, R. M. G. Perono, E. C. Amor, and W. L. Rivera, "Tannin-rich fraction from Terminalia catappa inhibits quorum sensing (QS) in Chromobacterium violaceum and the QS-controlled biofilm maturation and LasA staphylolytic activity in Pseudomonas aeruginosa," Journal of Ethnopharmacology, vol. 134, no. 3, pp. 865-871, 2011.

[32] M. D. Kiran, N. V. Adikesavan, O. Cirioni et al., "Discovery of a quorum-sensing inhibitor of drug-resistant staphylococcal infections by structure-based virtual screening," Molecular Pharmacology, vol. 73, no. 5, pp. 1578-1586, 2008.

[33] S. Sarabhai, P. Sharma, and N. Capalash, "Ellagic acid derivatives from Terminalia chebula Retz. downregulate the expression of quorum sensing genes to attenuate Pseudomonas aeruginosa PAO1 virulence," PLoS ONE, vol. 8, no. 1, Article ID e53441, 2013.

[34] V. Shukla and Z. Bhathena, "Sustained release of a purified tannin component of Terminalia chebula from a titanium implant surface prevents biofilm formation by Staphylococcus aureus," Applied Biochemistry and Biotechnology, vol. 175, no. 7, pp. 3542-3556, 2015.

[35] A. G. Al-Bakri and F. U. Afifi, "Evaluation of antimicrobial activity of selected plant extracts by rapid XTT colorimetry and bacterial enumeration," Journal of Microbiological Methods, vol. 68, no. 1, pp. 19-25, 2007.

[36] T. S. Roopashree, R. Dang, R. H. Shobha Rani, and C. Narendra, "Antibacterial activity of antipsoriatic herbs: Cassia tora, Momordica charantia and Calendula officinalis," International Journal of Applied Research in Natural Products, vol. 1, no. 3, pp. 20-28, 2008.

[37] A. E. Hagerman, Tannin Chemistry, 2011, http://www.users.miamioh.edu/hagermae/.

[38] K. H. McClean, M. K. Winson, L. Fish et al., "Quorum sensing and Chromobacterium violaceum: exploitation of violacein production and inhibition for the detection of $N$-acylhomoserine lactones," Microbiology, vol. 143, no. 12, pp. 3703-3711, 1997.

[39] C. Valgas, S. M. De Souza, E. F. A. Smânia, and A. Smânia Jr., "Screening methods to determine antibacterial activity of natural products," Brazilian Journal of Microbiology, vol. 38, no. 2, pp. 369-380, 2007.

[40] R. S. Blosser and K. M. Gray, "Extraction of violacein from Chromobacterium violaceum provides a new quantitative bioassay for N-acyl homoserine lactone autoinducers," Journal of Microbiological Methods, vol. 40, no. 1, pp. 47-55, 2000.

[41] R. J. C. Mclean, S. A. Bryant, D. A. Vattem, M. Givskov, T. B. Rasmussen, and N. Balaban, "Detection in vitro of quorumsensing molecules and their inhibitors," in Control of Biofilm Infections by Signal Manipulation, N. Balaban, Ed., vol. 2 of Springer Series on Biofilms, pp. 39-50, 2008.

[42] K. Mihalik, D. W. Chung, S. H. Crixell, R. J. C. Mclean, and D. A. Vattem, "Quorum sensing modulators of Pseudomonas aeruginosa characterized in Camellia sinensis," Asian Journal of Traditional Medicines, vol. 3, pp. 12-23, 2008.

[43] G. Ji, R. C. Beavis, and R. P. Novick, "Cell density control of staphylococcal virulence mediated by an octapeptide pheromone," Proceedings of the National Academy of Sciences of the United States of America, vol. 92, no. 26, pp. 12055-12059, 1995.

[44] A. Gupta, N. Tandon, and M. Sharma, Eds., Quality Standards of Indian Medicinal Plants Volume 2, Indian Council of Medical Research, New Delhi, India, 2005. 
[45] A. Gupta, N. Tandon, and M. Sharma, Eds., Quality Standards of Indian Medicinal Plants, vol. 1, Indian Council of Medical Research, New Delhi, India, 2003.

[46] A. Gupta, N. Tandon, and M. Sharma, Eds., Quality Standards of Indian Medicinal Plants, vol. 7, Indian Council of Medical Research, New Delhi, India, 2008.

[47] M. Daniel, Medicinal Plants: Chemistry and Properties, Science Publishers, 2006.

[48] C. Khare, Indian Herbal Remedies-Rational Western Therapy, Ayurvedic and Other Traditional Usage, Botany, Springer, Berlin, Germany, 2004.

[49] A. Gupta, N. Tandon, and M. Sharma, Eds., Quality Standards of Indian Medicinal Plants, vol. 8, Indian Council of Medical Research, New Delhi, India, 2010.

[50] V. Shukla and Z. Bhathena, "Crude extracts of Indian medicinal plants inhibit quorum sensing mediated by acyl homoserine lactones and auto inducing peptides," in Proceedings of the National Conference on Recent Trends and Future Prospects in Multidisciplinary Approaches in Microbiology (RTFPM '14), D. Nakade, Ed., pp. 145-152, Rajaram College, Kolhapur, India, 2014.

[51] H. S. Vasavi, A. B. Arun, and P. D. Rekha, "Inhibition of quorum sensing in Chromobacterium violaceum by Syzygium cumini L. and Pimenta dioica L.," Asian Pacific Journal of Tropical Biomedicine, vol. 3, no. 12, pp. 954-959, 2013.

[52] T. Okuda and H. Ito, "Tannins of constant structure in medicinal and food plants-hydrolyzable tannins and polyphenols related to tannins," Molecules, vol. 16, no. 3, pp. 2191-2217, 2011. 

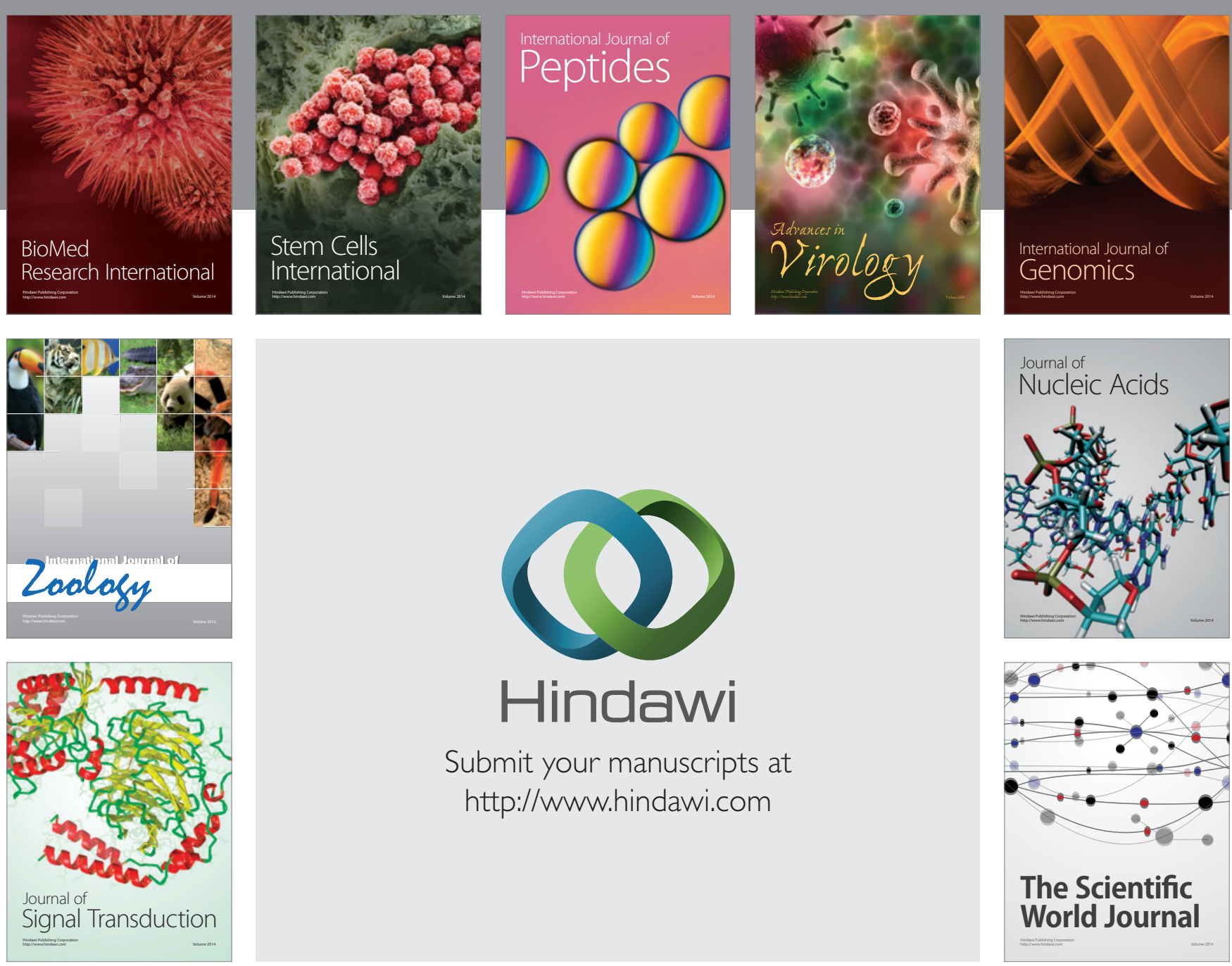

Submit your manuscripts at

http://www.hindawi.com
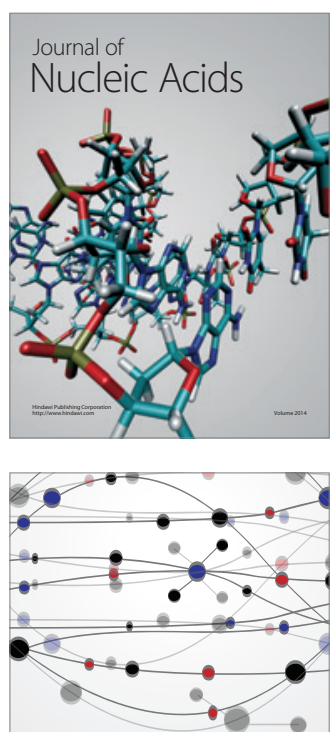

The Scientific World Journal
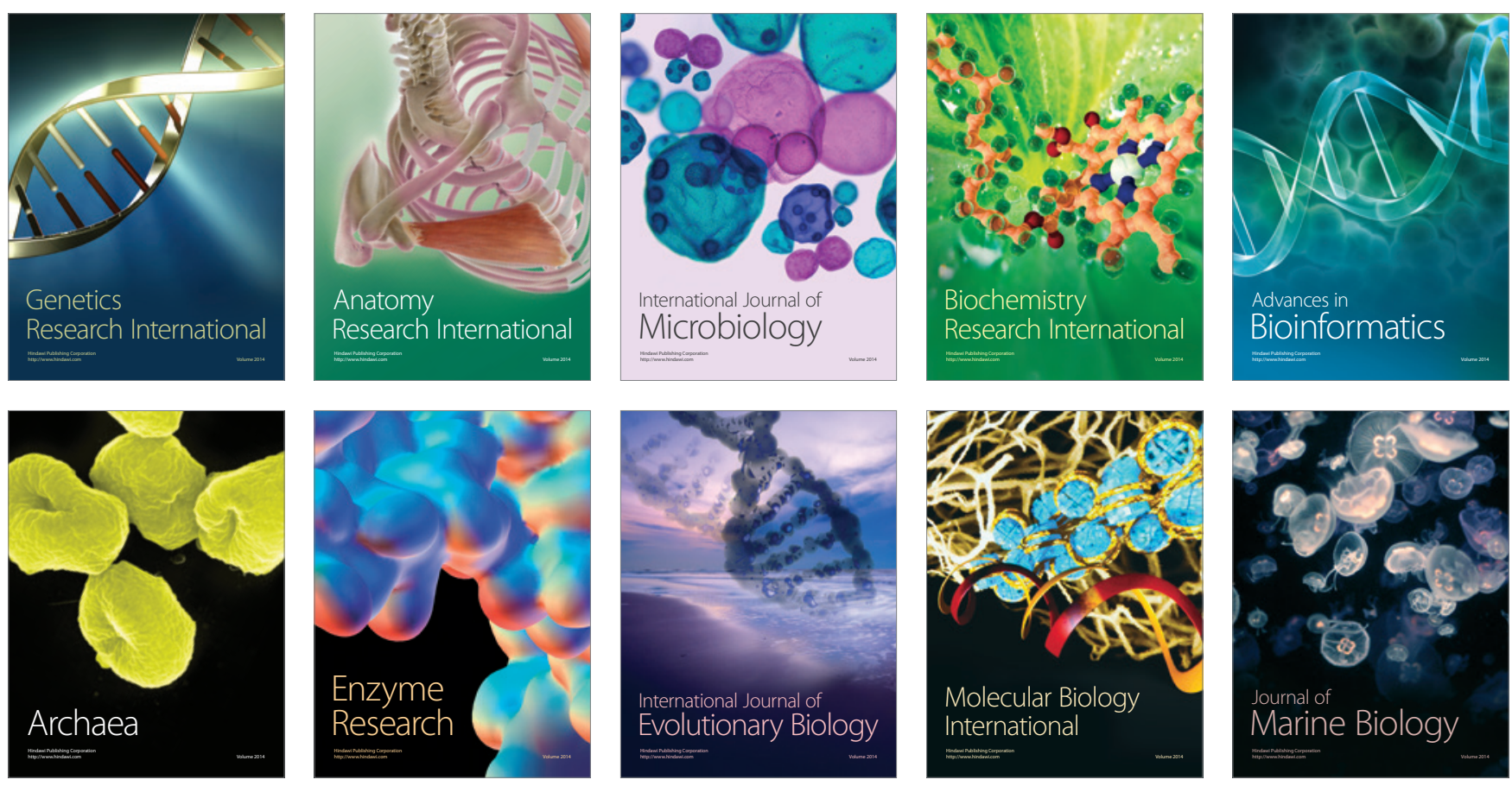